\title{
High-CHO diet increases post-exercise oxygen consumption after a supramaximal exercise bout
}

\author{
G.A. Ferreira ${ }^{1}$, R. Bertuzzi ${ }^{2}$, F.R. De-Oliveira ${ }^{3}$, F.O. Pires ${ }^{4}$ and A.E. Lima-Silva ${ }^{1}$ \\ ${ }^{1}$ Grupo de Pesquisa em Ciências do Esporte, Centro Acadêmico de Vitória, \\ Universidade Federal de Pernambuco, Vitória de Santo Antão, PE, Brasil \\ ${ }^{2}$ Grupo de Estudos em Desempenho Aeróbio da USP, Escola de Educação Física e Esporte, \\ Universidade de São Paulo, São Paulo, SP, Brasil \\ ${ }^{3}$ Núcleo de Estudos do Movimento Humano, Departamento de Educação Física, \\ Universidade Federal de Lavras, Lavras, MG, Brasil \\ ${ }^{4}$ Grupo de Estudos em Psico-fisiologia do Exercício, Escola de Artes, Ciências e Humanidades, \\ Universidade de São Paulo, São Paulo, SP, Brasi
}

\begin{abstract}
We investigated if carbohydrate $(\mathrm{CHO})$ availability could affect the excess post-exercise oxygen consumption (EPOC) after a single supramaximal exercise bout. Five physically active men cycled at $115 \%$ of peak oxygen uptake $\left(\mathrm{VO}_{2}\right.$ peak $)$ until exhaustion with low or high pre-exercise $\mathrm{CHO}$ availability. The endogenous $\mathrm{CHO}$ stores were manipulated by performing a glycogendepletion exercise protocol $48 \mathrm{~h}$ before the trial, followed by $48 \mathrm{~h}$ consuming either a low- $(10 \% \mathrm{CHO})$ or a high-CHO $(80 \%$ $\mathrm{CHO})$ diet regime. Compared to the low- $\mathrm{CHO}$ diet, the high- $\mathrm{CHO}$ diet increased time to exhaustion $(3.0 \pm 0.6 \mathrm{~min}$ vs $4.4 \pm 0.6$, respectively, $\mathrm{P}=0.01)$ and the total $\mathrm{O}_{2}$ consumption during the exercise $(6.9 \pm 0.9 \mathrm{~L}$ and $11.3 \pm 2.1$, respectively, $\mathrm{P}=0.01)$. This was accompanied by a higher EPOC magnitude $(4.6 \pm 1.8 \mathrm{~L}$ vs $6.2 \pm 2.8$, respectively, $\mathrm{P}=0.03)$ and a greater total $\mathrm{O}_{2}$ consumption throughout the session (exercise + recovery: $11.5 \pm 2.5 \mathrm{~L}$ vs $17.5 \pm 4.2$, respectively, $\mathrm{P}=0.01$ ). These results suggest that a single bout of supramaximal exercise performed with high $\mathrm{CHO}$ availability increases both exercise and postexercise energy expenditure.
\end{abstract}

Key words: Excess post-exercise oxygen consumption; EPOC; Supramaximal exercise; Energy expenditure; High-intensity exercise

\section{Introduction}

Exercise intensity is an important factor determining the excess post-exercise oxygen consumption (EPOC) (1). It has been described that there is a curvilinear relationship between EPOC magnitude (total $\mathrm{O}_{2}$ consumed during recovery) and exercise intensity (1). A larger EPOC [effect size $(E S)=0.9$ ] is observed after high-intensity exercise, compared to moderate-intensity ones, even when the total work performed is matched (2). The EPOC magnitude is also related to the exercise duration, in particular after a high-intensity, supramaximal exercise (SE) bout (3). For example, Bahr et al. (3) demonstrated that the EPOC magnitude was positively correlated to the exercise duration of an SE performed at $108 \%$ peak oxygen uptake $\left(\mathrm{VO}_{2}\right.$ peak $)$, once EPOC increased from 5.6 to $16.3 \mathrm{~L}$ $(E S=6.3)$ as the exercise duration increased from 2 to $6 \mathrm{~min}$. It is interesting to note that a single SE bout performed until exhaustion is considered a time-efficient alternative to increase post-exercise energy expenditure. This may have clinical applications because a single SE bout would increase post-exercise fat oxidation and insulin sensibility (4). Therefore, any manipulation that increases the time length of an SE bout will directly affect EPOC magnitude. This would be of practical relevance because a larger EPOC will increase post-exercise energy expenditure, which in turn could help individuals engaged in weight loss programs to reduce their body mass more rapidly and ameliorate their metabolic profile.

Regarding the main strategies to prolong SE duration, there are some evidences suggesting that high-carbohydrate $(\mathrm{CHO})$ availability has an important role (ES ranging from 0.7 to 2.4$)(5-7)$. We have previously demonstrated that the time to exhaustion during an SE was increased after $48 \mathrm{~h}$ under a high-CHO diet, when compared to an isocaloric, low-CHO diet $(E S=2.4)(6)$. This improvement in exercise tolerance with a high-CHO diet might be associated with several mechanisms such as an attenuation of

Correspondence: R. Bertuzzi: <bertuzzi@usp.br>

Received June 23, 2016 | Accepted August 30, 2016 


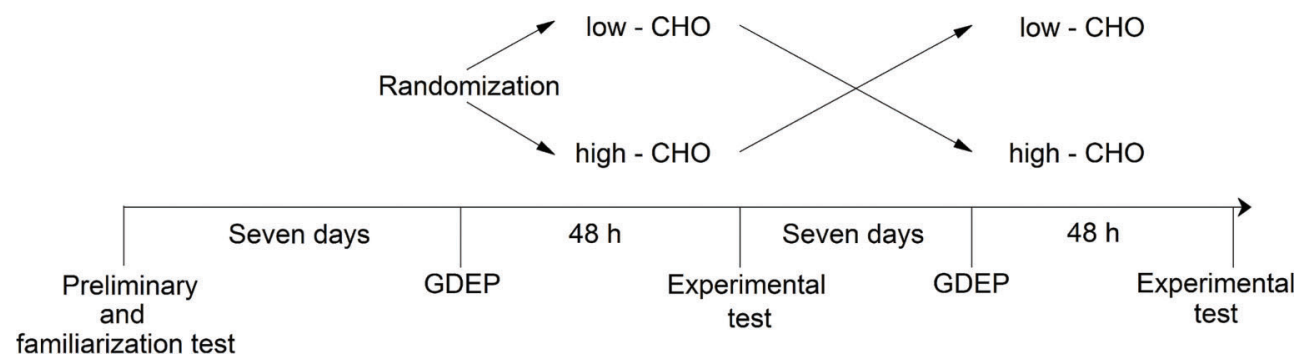

Figure 1. Experimental design. After the preliminary and familiarization test, and a 7-day period, participants were submitted to a glycogen-depletion exercise protocol (GDEP), followed by $48 \mathrm{~h}$ having either a high- or low- carbohydrate (CHO) diet. At the end of the 48-h period, participants returned to the laboratory and performed the test experiment for data collection. After a washout period of 7 days, the process was repeated with participants who had the high $\mathrm{CHO}$ diet previously, receiving the low $\mathrm{CHO}$ diet, and vice-versa.

central fatigue, a better maintenance of excitation-contraction coupling, a maintenance of $\mathrm{CHO}$ oxidation, and a reduced exercise-induced strain (8). In this regard, it could be hypothesized that the longer endurance time during an SE after a high-CHO diet period could lead to a greater EPOC and to a higher total energy expenditure in a given exercise training session, which could potentiate the body weight loss and reestablish metabolic balance in individuals engaged in training programs for health promotion. Thus, the interplay between SE duration and diet on postexercise metabolism is an important point to consider when making decisions about training prescription and establishing weight-loss approaches.

Therefore, we performed new analyses using a data set from a large project previously published (6) in order to determine the effects of a high-CHO diet on the EPOC after an SE bout performed until exhaustion. We hypothesized that the high-CHO diet would increase SE tolerance, thereby increasing EPOC compared to an isocaloric, low-CHO diet.

\section{Material and Methods}

\section{Participants}

The sample size calculation was based on the ES of a high- $\mathrm{CHO}$ diet on the SE duration and on the ES of SE duration on the EPOC, reported in Lima-Silva et al. (6) and Bahr et al. (3). The expected ESs from these studies were 2.4 and 4.0 , respectively. Thus, using the lowest ES (2.4), the minimum sample size required in this study for an alpha of 0.05 and a beta of 0.80 was 4 participants. Therefore, after receiving verbal and written explanations, and signing an informed consent, 5 physically active males (age $31.0 \pm 7.7$ years, height $180.2 \pm 4.3 \mathrm{~cm}$, body mass $77.0 \pm 7.7 \mathrm{~kg}$, body fat $13.3 \pm 2.9 \%, \mathrm{VO}_{2}$ peak $48.6 \pm 11.5 \mathrm{~mL} \cdot \mathrm{kg}^{-1} \cdot \mathrm{min}^{-1}$ ) volunteered to participate in this study, which was approved by the Ethics Committee of Universidade de São Paulo, SP, Brazil.

\section{Preliminary test and SE familiarization}

Participants completed an incremental test to exhaustion (starting at $50 \mathrm{~W}$ and increasing $20 \mathrm{~W}$ every $3 \mathrm{~min}$ ) on a cycle ergometer (Ergo Fit 167, Ergo-FitGmbH \& Co., Germany) to determine their first and second lactate thresholds ( $\mathrm{LT}_{1}$ and $\mathrm{LT}_{2}$, respectively) and $\mathrm{V} \mathrm{O}_{2}$ peak. Exhaustion was assumed when participants were unable to maintain a pedal cadence above $60 \mathrm{rpm}$. Blood micro-samples $(25 \mu \mathrm{L})$ were obtained at each stage from the earlobe and immediately analyzed for blood lactate concentration (La) [YSI 1500 Sport, Yellow Springs Instruments, USA]. The $\mathrm{VO}_{2}$ was continuously measured breath-by-breath (Quark b2, Cosmed, Italy). The blood La was plotted as a function of the workload, and the $\mathrm{LT}_{1}$ and $\mathrm{LT}_{2}$ were identified by a 3 -segment linear regression (9). The 3-segment linear regression fitting was done using an interactive process with two initially unknown intercepts calculated from every possible combination of intersections. The intercepts that best shared the curve in three linear segments were assumed when the highest $R^{2}$ value and the lowest residual sum of squares were attained. The $\mathrm{LT}_{1}$ was therefore defined as the workload corresponding to an initial change in the rate of lactate accumulation in the blood, while $\mathrm{LT}_{2}$ was defined as the workload corresponding to the second change in the rate of lactate accumulation. The $\mathrm{VO}_{2}$ peak was defined as the highest 30-s $\mathrm{VO}_{2}$ peak average at the end of the test.

Furthermore, in order to familiarize the participants with the experimental procedures, they performed an SE at $115 \%$ of $\mathrm{VO}_{2}$ peak until exhaustion, $48 \mathrm{~h}$ after the incremental test (Figure 1). At this intensity (100 to $120 \%$ $\dot{\mathrm{V}} \mathrm{O}_{2 \text { peak }}$ ), the exercise can be tolerated for a sufficient time to develop a reasonable EPOC, as previously reported $(3,10)$; time to exhaustion was also expected to be prolonged by the high- $\mathrm{CHO}$ diet in this exercise intensity, as reported elsewhere $(6,7)$.

\section{Experimental tests}

In order to produce a large difference in $\mathrm{CHO}$ availability, pre-SE endogenous $\mathrm{CHO}$ stores were altered by a combination of exercise and diet (11). First, participants performed a glycogen-depletion exercise protocol $48 \mathrm{~h}$ before each experimental session (12). This protocol consisted of a $90-$ min cycling at $50 \%$ of the difference between 
$\mathrm{LT}_{1}$ and $\mathrm{LT}_{2}$, followed by $6 \times 1 \mathrm{~min}$ exercise bouts at $125 \%$ of $\mathrm{VO}_{2 \text { peak }} ; 1$ min recovery was allowed between effort sets. After glycogen-depletion exercise protocol, participants followed an either high- or low- carbohydrate $(\mathrm{CHO})$ diet for $48 \mathrm{~h}$. At the end of the 48-h period, participants returned to the laboratory and performed the test experiment for data collection. After a washout period of 7 days, the process was repeated with participants who had the high $\mathrm{CHO}$ diet previously, receiving the low $\mathrm{CHO}$ diet, and vice-versa (crossover design, Figure 1).

In the test day, participants arrived at 8:00 am in the laboratory after a 12-h overnight fast and rested on a chair during 20 min for the assessment of resting $\mathrm{VO}_{2}$ value (Quark b2, Cosmed, Italy). Then, they underwent a 5-min warm-up at $50 \mathrm{~W}$, followed by an SE at $115 \%$ of $\mathrm{V} \mathrm{O}_{2}$ peak until exhaustion (6), which was assumed when participants were unable to maintain the pedal cadence above $60 \mathrm{rpm}$. Immediately after the test, they sat comfortably on a chair for 60 min. The $\mathrm{VO}_{2}$ peak was measured continuously from the baseline to the end of the 60 -min postexercise period (Quark b2, Cosmed).

\section{Calculations}

The rest $\mathrm{VO}_{2}$ peak of the last 1 min of the resting period was averaged and used as baseline. The total $\mathrm{O}_{2}$ consumed during the exercise was calculated as the area under the $\mathrm{VO}_{2}$-time curve, after deduction of the corresponding $\mathrm{VO}_{2}$ rest (13). EPOC duration (min) was considered as the time taken for the $\mathrm{VO}_{2}$ to return to baseline values (when the mean of five consecutive 1-min $\mathrm{VO}_{2}$ values were within $\pm 5 \%$ of the baseline $\mathrm{VO}_{2}$ (14). EPOC (L) was calculated as the integral of the $\mathrm{VO}_{2}$ versus time to reach the baseline minus the corresponding $\mathrm{VO}_{2}$ rest (15). All calculations were performed using the commercial software OriginPro 9.0 (OriginLab Corporation, USA). The total $\mathrm{O}_{2}$ required for the full session (exercise + recovery) was defined as the sum of the total $\mathrm{O}_{2}$ consumed during the exercise and EPOC. The total mechanical work performed was also calculated by multiplying external power output by exercise time duration.

\section{Statistical analysis}

Smirnov-Kolmogorov test was used for testing normality. After confirmation of normal distribution, a paired $t$-test was used to compare the exercise duration, exercise $\mathrm{O}_{2}$ consumption, EPOC duration, EPOC magnitude, and total $\mathrm{O}_{2}$ consumption between low- and high-CHO diet. Cohen's effect size was calculated and interpreted as proposed in (http://www.sportsci.org/resource/stats/): $\leqslant 0.2=$ trivial, $>0.2$ and $\leqslant 0.6=$ small, $>0.6$ and $\leqslant 1.2=$ moderate, and $>1.2=$ large. The power effect for each analysis is also reported. The level of significance was set at $P<0.05$. All statistical procedures were performed with Statistic software version 10 (StataSoft Inc. ${ }^{\circledR}$, USA).

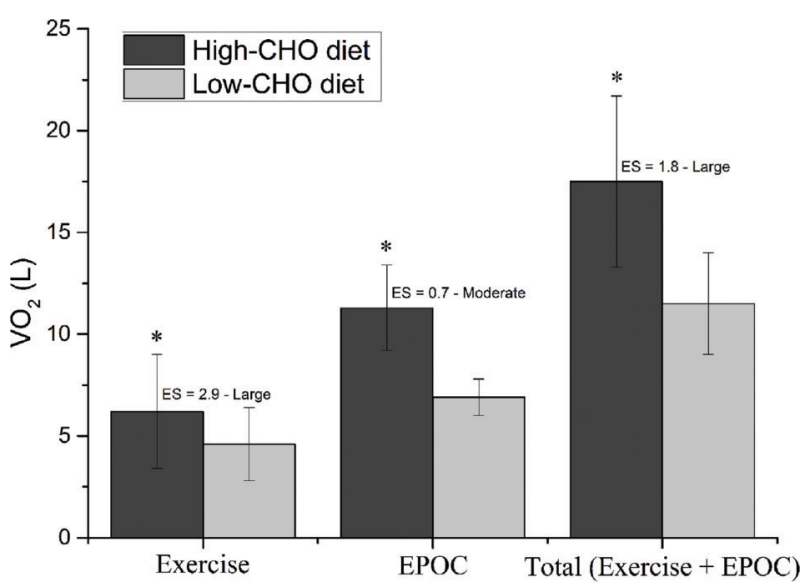

Figure 2. $\mathrm{O}_{2}$ consumption during exercise, post-exercise (excess post-exercise oxygen consumption, EPOC), and total oxygen demanded (exercise + recovery) in a supramaximal exercise bout performed until exhaustion, after pre-exercise carbohydrate $(\mathrm{CHO})$ manipulation. ES: effect size. Data are reported as means \pm SD. ${ }^{*} \mathrm{P}<0.05$ compared to low-CHO diet (paired $t$-test).

\section{Results}

The maximal power output $(\mathrm{PO})$ and $\mathrm{V} \mathrm{O}_{2 \text { peak }}$ reached during the incremental test were $251.2 \pm 36.2 \mathrm{~W}$ and $3.7 \pm 0.6 \mathrm{~L}$, respectively. The mean power output during the continuous exercise in the exercising-glycogen depletion protocol was $173.4 \pm 27.4 \mathrm{~W}(69.0 \pm 4.1 \%$ maximal power output during the incremental test). The supramaximal bouts of the exercising-glycogen depletion protocol (125\% $\mathrm{VO}_{2}$ peak $)$ were performed at $314.2 \pm 45.0 \mathrm{~W}$. The SE was performed at $289.0 \pm 41.3 \mathrm{~W}$.

The baseline $\mathrm{VO}_{2}$ was similar between high- and low$\mathrm{CHO}$ diet $(0.34 \pm 0.6$ vs $0.34 \pm 0.7 \mathrm{~L} / \mathrm{min}$, respectively, $\mathrm{P}=0.87$, $\mathrm{ES}=0.05$ trivial, power effect $=0.05$ ). There was a longer time to exhaustion $(4.4 \pm 0.6$ vs $3.0 \pm 0.6 \mathrm{~min}$, $\mathrm{P}=0.01, \mathrm{ES}=2.4$ large, power effect $=0.98$ ), and greater total mechanical work $(76.9 \pm 16.5$ vs $50.9 \pm 9.4 \mathrm{~kJ}$, $\mathrm{P}=0.001$, $E S=2.0$ large, power effect $=0.91$ ) and total $\mathrm{O}_{2}$ consumed $(11.3 \pm 2.1$ vs $6.9 \pm 0.9 \mathrm{~L}, \mathrm{P}=0.01$, $E S=2.9$ large, power effect $=0.99$ ) in high- than in low-CHO diet (Figure 2). The $\mathrm{VO}_{2}$ measured at exhaustion was slightly higher in high- compared to low-CHO diet (48.6 \pm 11.0 and $45.2 \pm 11.0 \mathrm{~mL} \cdot \mathrm{kg}^{-1} \cdot \mathrm{min}^{-1}$, respectively, $\mathrm{P}=0.004, \mathrm{ES}=0.3$ small, power effect $=0.08$ ).

The $\mathrm{VO}_{2}$ returned to baseline values within $60 \mathrm{~min}$ of rest in all participants and conditions. The EPOC duration was not significantly different between high- and low$\mathrm{CHO}$ diet $(30.0 \pm 12.4$ and $21.0 \pm 15.2 \mathrm{~min}$, respectively, $\mathrm{P}=0.16)$, although the $\mathrm{ES}$ was moderate ( $\mathrm{ES}=0.7$, power effect $=0.27)$. However, the EPOC magnitude was significantly increased in high- than in low- $\mathrm{CHO}$ diet $(6.2 \pm 2.8$ and $4.6 \pm 1.8 \mathrm{~L}$, respectively, $\mathrm{P}=0.03$, $\mathrm{ES}=0.7$ moderate, power effect=0.69, Figure 2). Consequently, the total $\mathrm{O}_{2}$ 
demanded (exercise + recovery) was greater in highcompared to low- $\mathrm{CHO}$ diet $(\mathrm{P}=0.01, \mathrm{ES}=1.8$ large, power effect $=0.98$, Figure 2).

\section{Discussion}

In the present study, we investigated the effects of low- and high- $\mathrm{CHO}$ diet on total $\mathrm{O}_{2}$ consumed during and after a SE bout performed until exhaustion. The exercise tolerance was significantly longer in high- than in low-CHO diet, thereby inducing a higher $\mathrm{VO}_{2}$ during the exercise as well as after the exercise. These results suggest that high$\mathrm{CHO}$ diet confers benefits considering exercise tolerance and energy expenditure during and after a single SE bout.

The high- $\mathrm{CHO}$ diet increased exercise duration ( 32\%) and total mechanical work $(\sim 34 \%)$ during a single SE bout. The increased tolerance further led to an increased exercise energy expenditure (i.e., $\sim 30 \% \mathrm{VO}_{2}$ increase). Previous studies had already demonstrated that energy expenditure during a high-intensity exercise is proportional to its duration (16). Although the anaerobic metabolism is highly demanded during high-intensity exercises, the additional energy expenditure necessary to support longer SE with high-CHO availability is probably covered by aerobic metabolism $(16,17)$. Although the mechanism for an increased SE tolerance with high- $\mathrm{CHO}$ availability is not fully understood, the assumption of elevated preexercise muscle glycogen stores seems to be improbable. Studies have shown that muscle glycogen is not fully depleted after a single SE bout performed until exhaustion (18), so that the remaining muscle glycogen would be sufficient to supply ATP and sustain a longer exercise. Therefore, fatigue during SE may be related to other factors such as a better metabolite balance, reduced exercise-induced strain and maintenance of excitationcontraction coupling (8). Additionally, the higher $\mathrm{CHO}$ availability can also play a central role by reducing the perceived effort during exercise, thus increasing the fatigue tolerance $(8,19,20)$.

Another interesting result of this study was that the longer SE found with the high-CHO diet was accompanied by a greater EPOC magnitude, which indicates elevated post-exercise energy expenditure. The EPOC magnitude has been attributed to several mechanisms such as beta adrenergic stimulation, triglyceride/fatty acids cycling, body temperature, increased lactate removal and oxidation, replenishment of muscle and blood oxygen stores, and creatine phosphate resynthesis $(1,21,22)$. Even though the shift from $\mathrm{CHO}$ to fat oxidation after the exercise bout with low-CHO availability may lead to an elevated EPOC $(1,23)$, in the present study the EPOC magnitude was $\sim 34 \%$ greater with high- $\mathrm{CHO}$ diet. This result suggests that the exercise duration during an SE bout is an important factor influencing EPOC magnitude $(1,14,24)$. Bahr et al. (3) demonstrated that EPOC increased 3 times ( $E S=6.3$ large) with a 3 times longer $S E$ (from 2 to $6 \mathrm{~min}$ ). A similar proportion has also been demonstrated by Hagberg et al. (25), who reported that EPOC increased 5 times when a high-intensity exercise ( $80 \%$ of $\mathrm{V} \mathrm{O}_{2 \text { peak }}$ ) was 4 times longer (from 5 to $20 \mathrm{~min}$ ). Interestingly, this $\sim 1: 1$ proportion and moderate to large ES persisted in the present study (i.e., 32\% longer exercise, 34\% larger EPOC magnitude, and large and moderate ES, respectively). In fact, longer exercise duration is thought to provoke larger metabolic disruption $(1,3,22,24-27)$, which might directly affect the return to homeostasis after the exercise. In this sense, prolonging the SE bout by $\sim 32 \%$ must have increased metabolic disturbance in the present study, and this may have been sufficient to proportionally increase EPOC magnitude ( 34\%).

Because exercise and post-exercise $\mathrm{VO}_{2}$ were increased, the total energy expenditure demanded in the training session (exercise + recovery) was $34 \%$ larger in high- than in low-CHO diet. This result suggests that combining high$\mathrm{CHO}$ diet and a SE bout until exhaustion could provide benefits considering energy expenditure. Recently, it has been debated that the lack of free time in modern life is a limiting factor for exercise practice and adherence (28-30). In this context, a short-duration SE bout until exhaustion has also been introduced as an alternative training model to stimulate active life style and to induce body weight reduction $(4,30)$. In a practical perspective, a few minutes of SE per day could increase daily energy expenditure. Interestingly, a single SE bout until exhaustion has been suggested to elicit similar health benefits compared to several intermittent bouts of SE with short intervals for recovery, despite the shorter duration of the former (4). Thus, our results indicate that a high- $\mathrm{CHO}$ diet seems to confer additional benefits regarding the total energy expended during a SE bout performed until exhaustion. It would be interesting to investigate in further studies the accumulative effect of combining a high- $\mathrm{CHO}$ diet and training with a single exhaustive bout of SE on energy expenditure, weight loss and metabolic adaptations.

The main limitation of the present study is the small sample size. However, in an attempt to minimize possible bias, we calculated the Cohen's ES between conditions for time to exhaustion, total $\mathrm{O}_{2}$ consumed, EPOC magnitude and total $\mathrm{O}_{2}$ demanded (exercise + recovery). Despite the small sample size, the statistical power ranged from 0.69 to 0.98 for total $\mathrm{O}_{2}$ consumed, EPOC magnitude and total $\mathrm{O}_{2}$ demanded (exercise + recovery). Therefore, the sample size was enough to achieve a satisfactory power effect, which in turn avoids a type II error. In addition, we are not able to extrapolate our results to a longitudinal perspective; further studies investigating the effect of combining a high- $\mathrm{CHO}$ diet and SE on energy expenditure and metabolic balance using a longitudinal-intervention design, are required.

In summary, when compared to a low-CHO diet, a high- $\mathrm{CHO}$ diet leads to a higher exercise tolerance, and 
higher consumed oxygen during and after a single exhaustive SE bout. From a practical standpoint, this might be an

\section{References}

1. Borsheim E, Bahr R. Effect of exercise intensity, duration and mode on postexercise oxygen consumption. Sports Med 2003; 33: 1037-1060, doi: 10.2165/00007256-200333140-00002.

2. Larsen I, Welde B, Martins C, Tjonna AE. High- and moderateintensity aerobic exercise and excess post-exercise oxygen consumption in men with metabolic syndrome. Scand J Med Sci Sports 2014; 24: e174-e179, doi: 10.1111/sms.12132.

3. Bahr R, Gronnerod O, Sejersted OM. Effect of supramaximal exercise on excess postexercise $\mathrm{O}_{2}$ consumption. Med Sci Sports Exerc 1992; 24: 66-71, doi: 10.1249/00005768199201000-00012.

4. Whyte LJ, Ferguson C, Wilson J, Scott RA, Gill JM. Effects of single bout of very high-intensity exercise on metabolic health biomarkers in overweight/obese sedentary men. Metabolism 2013; 62: 212-219, doi: 10.1016/j.metabol. 2012.07.019.

5. Lima-Silva AE, De-Oliveira FR, Nakamura FY, Gevaerd MS. Effect of carbohydrate availability on time to exhaustion in exercise performed at two different intensities. Braz $\mathrm{J}$ Med Biol Res 2009; 42: 404-412, doi: 10.1590/S0100-879X2009 000800008.

6. Lima-Silva AE, Pires FO, Bertuzzi R, Silva-Cavalcante MD, Oliveira RS, Kiss MA, et al. Effects of a low- or a highcarbohydrate diet on performance, energy system contribution, and metabolic responses during supramaximal exercise. Appl Physiol Nutr Metab 2013; 38: 928-934, doi: 10.1139/ apnm-2012-0467.

7. Lira FS, Zanchi NE, Lima-Silva AE, Pires FO, Bertuzzi RC, Caperuto EC, et al. Is acute supramaximal exercise capable of modulating lipoprotein profile in healthy men? Eur J Clin Invest 2010; 40: 759-765, doi: 10.1111/j.1365-2362.2010.02316.x.

8. Karelis AD, Smith JW, Passe DH, Peronnet F. Carbohydrate administration and exercise performance: what are the potential mechanisms involved? Sports Med 2010; 40: 747-763, doi: 10.2165/11533080-000000000-00000.

9. Ribeiro JP, Yang J, Adams RP, Kuca B, Knutten HG. Effect of different incremental exercise protocols on the determination of lactate and ventilatory thresholds. Braz J Med Biol Res 1986; 19: 109-117.

10. Zanconato S, Cooper DM, Armon Y. Oxygen cost and oxygen uptake dynamics and recovery with 1 min of exercise in children and adults. J Appl Physiol 1991; 71: 993-998.

11. Hargreave M, McconelL G, Proieto J. Influence of muscle glycogen on glycogenolysis and glucose during exercise in humans. J Appl Physiolo 1985; 78: 288-292.

12. Heigenhauser GJ, Sutton JR, Jones NL. Effect of glycogen depletion on the ventilatory response to exercise. $J$ Appl Physiol Respir Environ Exerc Physiol 1983; 54: 470-474.

13. Artioli GG, Bertuzzi RC, Roschel H, Mendes SH, Lancha AH $\mathrm{Jr}$, Franchini $\mathrm{E}$. Determining the contribution of the energy systems during exercise. J Vis Exp 2012, doi: 10.3791/3413.

14. LaForgia J, Withers RT, Gore CJ. Effects of exercise intensity and duration on the excess post-exercise oxygen consumption. J Sports Sci 2006; 24: 1247-1264, doi: 10.1080/02640410600552064. appealing strategy for a less time-consuming training and weight loss.

15. Segal SS, Brooks GA. Effects of glycogen depletion and work load on postexercise $\mathrm{O}_{2}$ consumption and blood lactate. J Appl Physiol Respir Environ Exerc Physiol 1979; 47: 514-521.

16. Poole DC, Ward SA, Gardner GW, Whipp BJ. Metabolic and respiratory profile of the upper limit for prolonged exercise in man. Ergonomics 1988; 31: 1265-1279, doi: 10.1080/ 00140138808966766.

17. Bogdanis GC, Nevill ME, Boobis LH, Lakomy HK. Contribution of phosphocreatine and aerobic metabolism to energy supply during repeated sprint exercise. J Appl Physiol 1996; 80: 876-884.

18. Vandenberghe K, Hespel P, Vanden EB, Lysens R, Richter EA. No effect of glycogen level on glycogen metabolism during high intensity exercise. Med Sci Sports Exerc 1995; 27: 1278-1283, doi: 10.1249/00005768-199509000-00007.

19. Ferreira GA, Osiecki R, Lima-Silva AE, de Angelis-Pereira $\mathrm{MC}$, De-Oliveira FR. Effect of a reduced-CHO diet on the rate of perceived exertion curve during an incremental test. Int J Sport Nutr Exerc Metab 2014; 24: 532-542, doi: 10.1123/ijsnem.2013-0248.

20. Steiner JL, Curmaci A, Patrie JT, Gaesser GA, Weltman A. Effects of carbohydrate supplementation on the RPE-blood lactate relationship. Med Sci Sports Exerc 2009; 41: 13261333, doi: 10.1249/MSS.0b013e3181967637.

21. Gaesser GA, Brooks GA. Metabolic bases of excess postexercise oxygen consumption: a review. Med Sci Sports Exerc 1984; 16: 29-43.

22. Borsheim E, Bahr R, Hostmark AT, Knardahl S. Effect of beta-adrenoceptor blockade on postexercise oxygen consumption and triglyceride/fatty acid cycling. Metabolism 1998; 47: 439-448, doi: 10.1016/S0026-0495(98) 90057-9

23. Frost EA, Redman LM, de Jonge L, Rood J, Zachwieja JJ, Volaufova J, et al. Interaction between dietary fat and exercise on excess postexercise oxygen consumption. Am J Physiol Endocrinol Metab 2014; 306: E1093-E1098, doi: 10.1152/ajpendo.00383.2013.

24. Bahr R, Ingnes I, Vaage O, Sejersted OM, Newsholme EA. Effect of duration of exercise on excess postexercise O2 consumption. J Appl Physiol 1987; 62: 485-490, doi: 10.1063/1.339771.

25. Hagberg JM, Mullin JP, Nagle FJ. Effect of work intensity and duration on recovery $\mathrm{O}_{2}$. J Appl Physiol Respir Environ Exerc Physiol 1980; 48: 540-544.

26. Gore CJ, Withers RT. Effect of exercise intensity and duration on postexercise metabolism. J Appl Physiol 1990; 68: 2362-2368.

27. Chad KE, Wenger HA. The effect of exercise duration on the exercise and post-exercise oxygen consumption. Can J Sport Sci 1988; 13: 204-207.

28. Trost SG, Owen N, Bauman AE, Sallis JF, Brown W. Correlates of adults' participation in physical activity: review and update. Med Sci Sports Exerc 2002; 34: 1996-2001, doi: 10.1097/00005768-200212000-00020. 
29. Little JP, Safdar A, Wilkin GP, Tarnopolsky MA, Gibala MJ. A practical model of low-volume high-intensity interval training induces mitochondrial biogenesis in human skeletal muscle: potential mechanisms. J Physiol 2010; 588: 10111022, doi: 10.1113/jphysiol.2009.181743.
30. Gillen JB, Percival ME, Skelly LE, Martin BJ, Tan RB, Tarnopolsky MA, et al. Three minutes of all-out intermittent exercise per week increases skeletal muscle oxidative capacity and improves cardiometabolic health. PLoS One 2014; 9: e111489, doi: 10.1371/journal.pone.0111489. 